\title{
ANT-LION BASED EFFICIENT COGNITIVE RADIO ROUTING
}

\author{
Anshu $^{1} \&$ Vinod Kumar Srivastava ${ }^{2}$
}

Abstract- This paper designs an efficient routing technique for the cognitive radio by using a latest optimized meta-heuristic approach, Ant-lion optimization. Ant-Lion hunting behaviour is implemented in the Ant Lion optimization (ALO) algorithm. The Ant lion traps the ant given after the random movement of the ant. This phenomenon is used in cognitive radio to access the maximum channel bandwidth irrespective to the channel variations. The algorithm is compared against three state of art techniques, Ant based cognitive radio, chain based cognitive radio and efficient cognitive radio.

Keywords: Cognitive Radio, Ant lion, Ant, Ant lion optimization, Meta-heuristic

\section{INTRODUCTION}

Cognitive Radio (CR) is a worldview for remote correspondence in which either a system or a remote hub changes its transmission or gathering parameters to discuss effectively maintaining a strategic distance from obstruction with authorized or unlicensed users[1,2,3]. This adjustment of parameters depends on the dynamic checking of a few factors in the outside and interior radio condition, for example, radio recurrence range, client conduct, and system state [4,5,6,8]. CRs have two principle qualities: Cognitive capacity that makes these gadgets fit for detecting their condition and picking the best accessible transmission mode (e.g. adjustment sort) in the decrepit groups. This ends up plainly practical through the range administration handle where a few physical layer parameters, for example, recurrence, adjustment sort, control, and so forth., are assessed [2,7]. Reconfigurability that empowers a CR to change a few of its parameters (e.g. recurrence, balance, and so forth.) and adjust to its condition. This is critical as CRs should utilize the neglected groups in a deft way, clearing a band (through range hand-off) if any PU transmissions are recognized. CRs organization ends up noticeably achievable using the SDRs a significant number of which are outfitted with gadgets with reprogrammable components (e.g. field programmable door exhibits or universally useful processors) that can change their physical layer parameters on-the-fly.

\subsection{Cognitive Capability:}

As said in the past areas, CR is a gadget that can detect its condition and adjust as needs be. The operations that a CR performs for versatile operation are alluded to as the subjective cycle, appeared in Figure 1: (the circles appeared on this figure). SUs get to any of the accessible range gaps astutely. On the off chance that an occupant flag is recognized, SUs need to abandon the particular band instantly. The psychological cycle comprises of the accompanying mechanisms[9]:

\subsection{Spectrum detecting:}

Officeholder signs' location is performed amid range detecting that is a standout amongst the most essential segments of a CR [1]. For instance, in IEEE 802.22, there are quiet periods, in which all SUs abstain from transmission so as to perform range detecting for the discovery of officeholder signals.

\section{RECONFIGURABILITY}

Reconfigurability is an essential characteristic of CRs that is closely related to their cognitive capability. Reconfiguration of CRs involves the change/modification/update of several characteristics of their physical layer parameters such as: (i) carrier frequency, (ii) type of modulation, (iii) transmission power, etc. CRs have to be flexible and adapt to the environment; therefore physical layer mechanisms purely implemented at the hardware level are not sufficient. SDRs are a viable solution to this concern. These are devices with radio functionality modules implemented in software, providing reconfigurability by using the same equipment in different regions and under different policies (e.g. spectrum regulation enforced by the authorities). In general, a SDR consists of two distinct parts: hardware and software, as shown in Figure 2 Furthermore, the software part consists of several sub-modules:

\footnotetext{
${ }^{1}$ Research Scholar, Department of computer science, Baba Mastnath University, Rohtak

${ }^{2}$ Professor, Department of computer science, Baba Mastnath University, Rohtak
} 


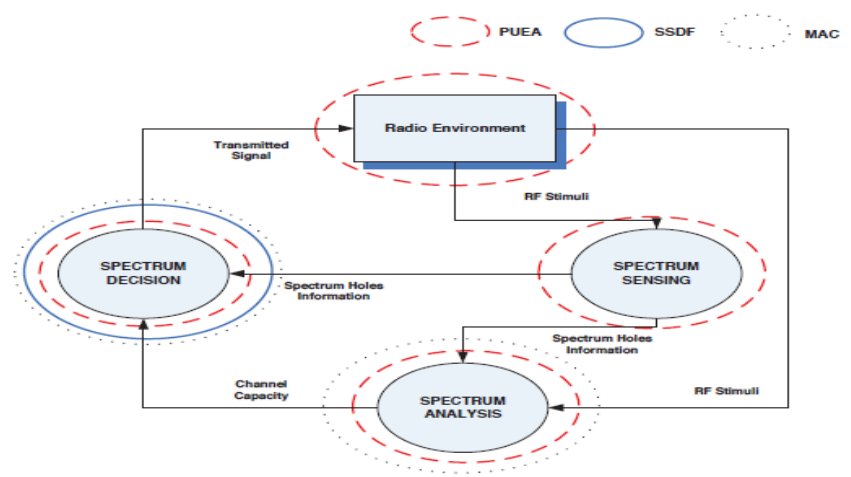

Figure 1: The Cognitive Cycle

- Radio operation environment (ROE) contains all the core modules for the radio configuration (e.g. driver, middleware, operating system).

- Radio applications (RA) controls the functionality of the radio platform, implementing the air interference and the communication protocols.

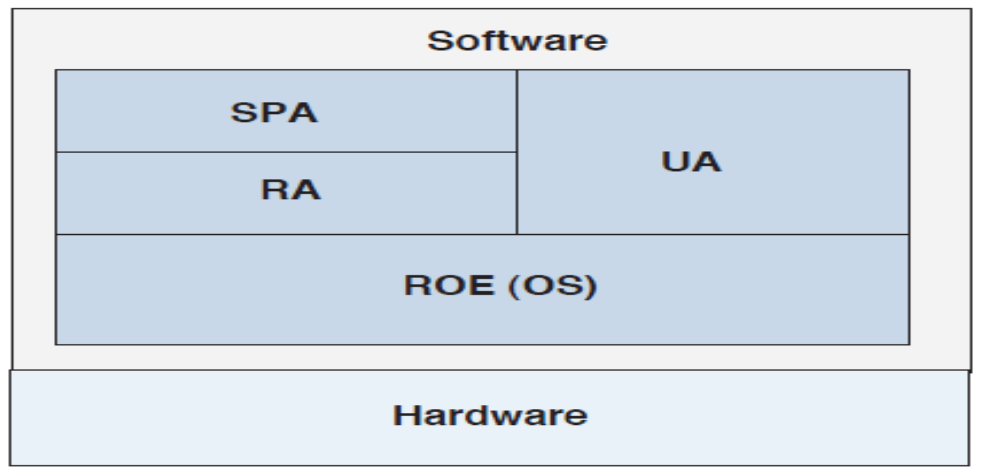

Figure 2: Software-Defined Radio Architecture

- Service provider applications (SPA) include services such as messaging, video, voice, etc.

- User applications (UA) include all the applications installed by a user (e.g. text editors, web browsers, etc.

\section{EXISTING WORK}

The Chain based Routing conventions depicted for Cognitive radio senor systems (CRC). The sensor arrange involved quantities of asset obliged sensor hubs. The sensor hubs are battery controlled. Sent sensor hubs are homogenous as far as equipment and beginning battery control which is the vitality source. After some time, the sensor hubs might be forgotten with non-uniform level of vitality. The base station is found far from the sensor field. The base station is an ingenious hub and vitality is not a limitation for the base station. The sensor hubs transmit information bundles towards the base station in unicast mode. It is expected that the base station has a substantial transmission range and it can transmit specifically even to the most distant hub. By and large regular WSN can't utilize unused range in other band and it works statically specifically direct in unlicensed band. Be that as it may, subjective radio sensor organize (CRSN) can work in various channels of unlicensed and also authorized groups. CSRN can exist together with heritage systems. The clients of a specific channel are delegated Primary User (PU) and Secondary User (SU). The PUs have higher need over SUs for utilizing the individual channels. In this work, whatever other system application other than CR empowered systems are considered as PUs and CR empowered systems, for example, CRSNs are considered as SUs. The two PUs and SUs are relied upon to coincide [5]. The current convention is examined underneath as far as five stages. These are confinement, synchronization, chain arrangement, vitality adjusted information sending, and renewal of chains. Each of these stages is examined in detail beneath.

\subsection{Localization:}

The sensor hubs are to be related to regard to their geographic areas. The base station isolates the sensor field regarding areas as appeared in the Figure3. There are two unique parameters relying upon which the areas are framed. These are: the point of transmission/gathering of signs $(\theta)$ by the base station and the transmission range of the base station (r). Subsequently every segment is spoken to by two parameters, i.e., the traverse ( $\theta \mathrm{i}$ to $\theta \mathrm{j})$ and the transmission sweep run (ra to $\mathrm{rb}$ ). Once the parts are framed as appeared in the figure 3 by the base station, every sensor hub is relegated a proper area number. The base station 
can dole out a proper division number to every sensor hub, once the base station gets no less than one flag from every sensor hub. The edge of gathering of the flag by the base station and the relating signal quality are utilized together to figure the best possible division number for every sensor hub. Every sensor hub in the field is restricted through this division numbers and the relative places of various sensor hubs are processed. Toward the finish of this stage the base station finds each of the sensor hubs sent in the field. This area data of a sensor hub is a relative amount. The base station does not have to take help of any GPS (Geographic Position System) based procedures keeping in mind the end goal to find the sensor hubs. In addition, the sensor framework under thought does not require the real area data of every sensor hub that regularly is achievable through GPS based framework. The relative places of the sensor hubs are adequate for finish limitation of the hubs in the sensor organize keeping in mind the end goal to settle on the steering choices [5].

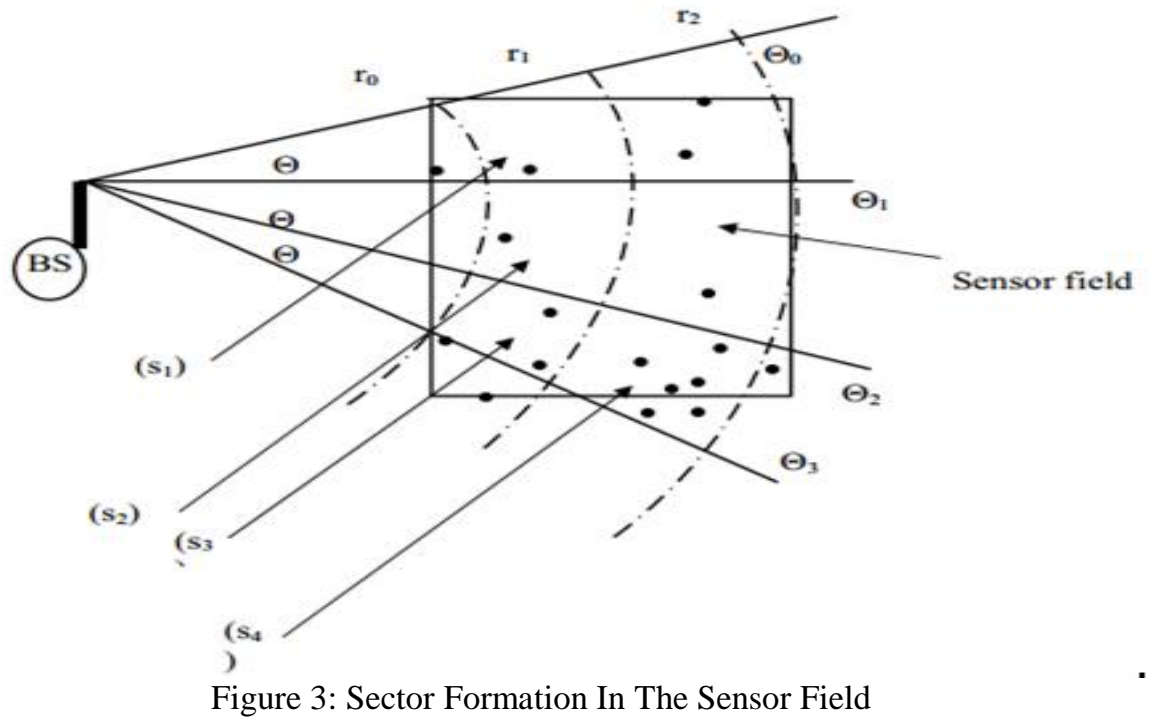

\subsection{Synchronization:}

Figure 3: Sector Formation In The Sensor Field

The sensor nodes are necessary to be synchronized with the base station. Since the sensor nodes are going to share different frequency bands, it is important for the base station to know the local time of different sensor nodes and they need to communicate in a synchronized manner. This feature becomes even more important for supporting real time communication. The base station and the sensor nodes can be synchronized by executing some existing standard synchronization algorithm. Most of such algorithms involve few rounds of communication between the sensor nodes and the base station. Simple and lightweight synchronization algorithms are appropriate to apply for synchronization for resource constrained sensor nodes.

\subsection{Chain formation:}

The base station organizes the sensor nodes in terms of different chains. Each chain starts from a certain node and ends at the base station. Any node interested to forward data to the base station does so, by using other intermediate nodes present in the chain connecting the sender node and the base station. The nodes constituting each chain are selected by the base station considering different parameters.

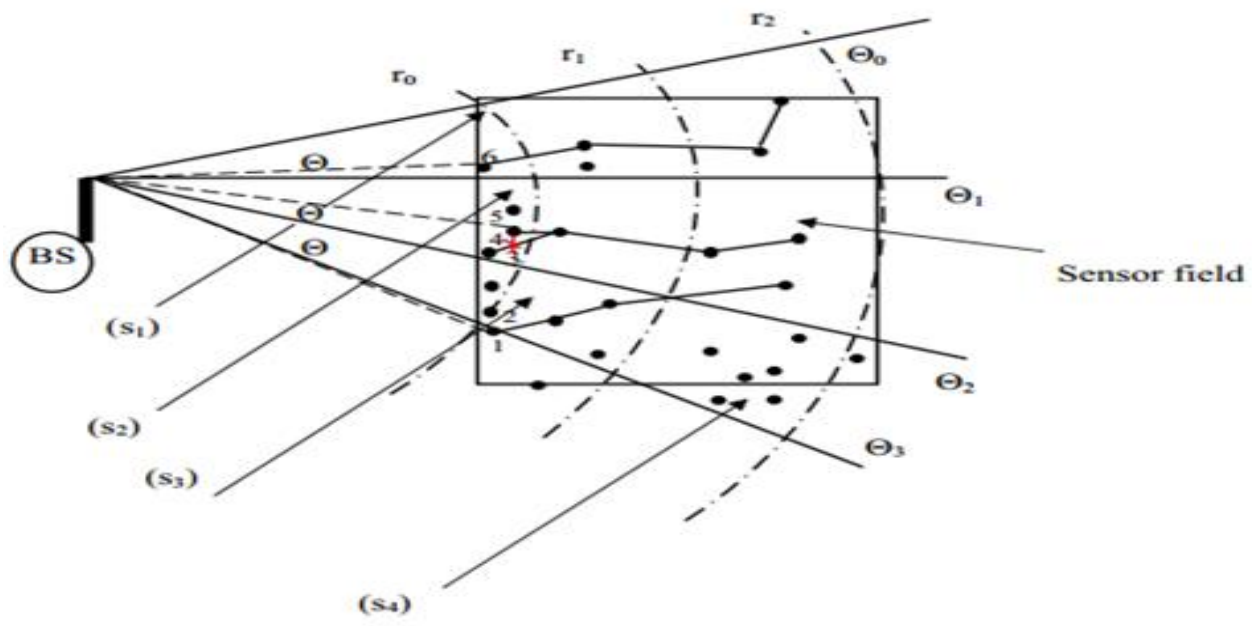

Figure 4: Chain Formation through Different Sectors In The Sensor Field 
The chains are formed in such a way that each node is present exactly in one chain only. Once chain formation phase is over the entire sensor field can be visualized as a set of chains. Figure 4 shows an instance of the sensor field after chain formation. The cross in Figure 4 indicates the cancellation of the link otherwise that link may make the node 10 present in two different chains. Whenever a node needs to forward data to the base station, it gets a suitable chain selected by the base station as per the selection rules. Different selection rules along with different parameters are discussed below.

Each chain $C$ can be considered as a set of vertices $(V)$ and edges $(E)$, thus $C=(V, E)$. Moreover, no two chains (say $C_{i}$ and $C_{j}$ ) can have common vertices and edges, thus $\mathrm{C}_{\mathrm{i}} \cap \mathrm{C}_{\mathrm{j}}=\phi$. There are several parameters considered by the base station while identifying different nodes for the chains. These are enlisted below:

Residual energy level: Node with higher residual energy is preferred in order to form the chains. Location information: The location information i.e., the sector information of each candidate node is considered for identifying the nodes for different chains. This information is helpful for identifying the next node in a chain. Normally, only one node from one sector remains present in a chain. The next node in the chain belongs to any one of the adjacent sectors. Channel commonality: While the routes are selected, it is taken into consideration that a route should have more numbers of common channels. The routes with more numbers of common channels are preferred over the routes with less number of common channels. This is so because channel switching is a source of energy expenditure. Frequent channel switching is to be avoided in order to save energy expenditure. Here the terms route and chain are used interchangeably. Thus the nodes for a chain are selected considering the channel commonality as one of the parameters.

Number of hops: Number of hops in a route should be less in numbers. Minimum numbers of hops reduces energy consumption in a route. Availability of licensed channel: The presence of licensed channel in a route makes it possible to have long distance transmission. It also reduces frequent channel switching. Thus a route that has licensed channel available is preferable.

\subsection{Energy balanced data forwarding:}

Since some sensor nodes need to forward data of other nodes as well, the energy available in those intermediate nodes may drain out quickly if they happen to forward data of other nodes

heavily and frequently. On the other hand, depending on the data transmission patterns of different nodes, some chains of nodes may be lightly loaded with data traffic. Then these chains of node will naturally live long. Such imbalance in energy consumption among the nodes is not encouraged. Moreover, if a chain of nodes involves more number of frequency bands switching than other chains then it is highly probable that the nodes in such a chain will exhaust their energy earlier than other nodes. Therefore, the data is to be forwarded towards the base station maintaining a balance of load among different chains of nodes available in the field. It is assumed that the base station has an idea about the primary (PU) and secondary users (SU) of a channel. Depending on the availability of channels, the base station instructs the sensor nodes to forward data through differrent channels at different time slots. The base station is a resourceful computing node and therefore, most of the computing burdens are shifted towards the base station.

\subsection{Re-formation of chains:}

If some nodes present in different chains die out then the routes break. This has significant impact on the performance of the protocol in terms of data delivery. At this stage the chains are to be formed again. The base station takes initiatives and reframes the chains among the available nodes again by calling the same procedure.

\section{ANT LION OPTIMIZATION (ALO)}

Ant lion algorithm (ALO) exhibits the hunting behavior of the Ant lion also known as doodlebugs. Ant lion attacks the ant in strategic manner by building the traps which is explained in the ant lion algorithm. The ALO completes its processing in five steps i.e. random movements of the ant, building traps by the ant lion, entrapment of ant in to traps designed by ant lion, catching prey i.e. ant and rebuilding of the trap with better fitness. These five phases completes the process of ALO elaborated below:

Random Movements of the Ant: Each ant moves in a random direction and the other ants follow it by the pheromone generated by the ant. This section describes the ant lion- optimization algorithm as follow: In the ALO, $\mathrm{n}$ ant lions are travelling towards the ant in dimensions then the position matrix can be given shown as eq. 1 .

$$
P_{m}=\left[\begin{array}{ccc}
P_{1,1} & \cdots & P_{1, d} \\
\vdots & \ddots & \vdots \\
P_{n, 1} & \cdots & P_{n, d}
\end{array}\right]
$$

The best position in the matrix of equation (1) can be found by analyzing the corresponding values in fitness function (best solution can be minimum or maximum value depending upon the problem). The fitness function can be given as (2)

$$
F_{v_{-} f}=\left[\begin{array}{c}
F_{v_{-} f 1} \\
\vdots \\
F_{v_{-} f n}
\end{array}\right]
$$


When the ant lion reaches nearer to the ant then the ant lion starts to follow the spiral motion which can be specified by using eq.(5) as shown:

$P_{m_{i}, f_{j}}=F_{v_{-} f j}+d * \cos (2 \pi r) * e^{b r}$

The $P_{m i, f_{j}}$ denotes the updated position using the spiral motion while the $\mathrm{b}$ is a constant to define shape of the motion. The $\mathrm{r}$ is used to define the ant lion and relationship if the value of $r$ is 1 then the ant lion follows the , resulting the best possible position near the is selected otherwise the ant lion selects the best far position from the This updated position is used to move towards the optimization. The distance $\mathrm{d}$ is given by eq.(4).

$\mathrm{d}=\left|F_{v_{-} f j}-F_{v_{-} m i}\right|$

The above algorithm describes the ALO algorithm. The next section discusses the implementation of ALO in our work.

\section{OPTIMIZION BASED ON ANT LION ALGORITHM}

In this work the algorithm is optimized by using the ant lion algorithm. The ant lion are replaced by the nodes i.e. there is one ant lion for each node and the position of the node is considered as the position of the ant lion. The position matrix representing the ant lion based on nodes is given by eq. (5).

$M_{N}=\left[\begin{array}{ccc}M_{N_{1,1}} & M_{N_{1,2}} & M_{N_{1, n}} \\ M_{N_{2,1}} & M_{N_{2,2}} & M_{N_{2, n}} \\ \vdots & \vdots & \vdots \\ M_{N_{n, 1}} & M_{N_{n, 2}} & M_{N_{n, n}}\end{array}\right]$

Here, $\mathrm{n}$ is the number of nodes in the network. The distance of current node from the destination is used to generate the corresponding fitness value. The fitness value is generated by using the eq. (6).

$$
M_{N_{1}}=\sqrt{\left(M_{N_{d, 1}}-M_{N_{1,1}}\right)^{2}+\left(M_{N_{d, 2}}-M_{N_{1,2}}\right)^{2}+\left(M_{N_{d, 3}}-M_{N_{1,3}}\right)^{2}}
$$

Corresponding fitness value matrix is given by the equation (7):

$O_{M_{N}}=\left[\begin{array}{c}O_{M_{N_{1}}} \\ \vdots \\ O_{M_{N_{n}}}\end{array}\right]$

The matrix gives the position matrix of the best neighbor node to be selected in the route by any node. The complete process can also be understood by the following algorithm:

Algorithm( Source, Destination)

1. Initialize the Network:

For $\mathrm{i}=1: \mathrm{N}$

For $\mathrm{j}=1: \mathrm{N}$

End

$\mathrm{M}_{\mathrm{Ni}, \mathrm{j}}=$ random position between given network area

Calculate fitness value

$M_{N_{i}}=\sqrt{\left(M_{N_{d, i 1}}-M_{N_{i, 1}}\right)^{2}+\left(M_{N_{d, 2}}-M_{N_{i, 2}}\right)^{2}+\left(M_{N_{d, 3}}-M_{N_{i, 3}}\right)^{2}}$

End

2. $\quad \mathrm{F}_{\mathrm{N}}=\operatorname{Best}\left(\mathrm{M}_{\mathrm{N}}\right)$

3. $\mathrm{O}_{\mathrm{FN}}=\operatorname{Best}\left(\mathrm{O}_{\mathrm{MN}}\right)$

4. Current $_{\text {node }}=$ Source

5. While Current $_{\text {node }} \neq$ Destination

i. $\quad d=\left|F_{j}-M_{i}\right|$

ii. $\quad M_{M_{i,} F_{j}}=d * e^{b t} * \cos (2 \pi t)+F_{j}$

iii. Generate the Distance matrix by using eq. (6)

iv. Select Next Node in The route

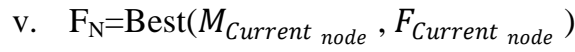

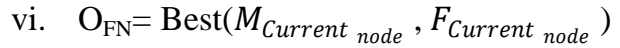

vii. End if

viii. Update $b$ and $t$ End while

ix. Update Current node

6. Exit 
The above algorithm is used to generate an optimized path. The implementation and results of the algorithm are discussed in next section.

\section{RESULTS}

The modified chain based cognitive radio is compared with existing chain based cognitive radio protocol using various parameters that are explained below.

1. Energy Consumed: It is amount of energy consumed for transferring the data from source to destination. The mean of energy of energy consumed by each node is the average energy consumption in the network. $\sum$ Energy consumed by each node/number of nodes

2. Residual Energy: It is the difference of total energy and the energy consumed by the network.

$\sum$ Energy of each node - $\sum$ energy consumed by each node

3. Network Life time: It is the time until all nodes within the network get dead. In other words, it is the operating time of the network.

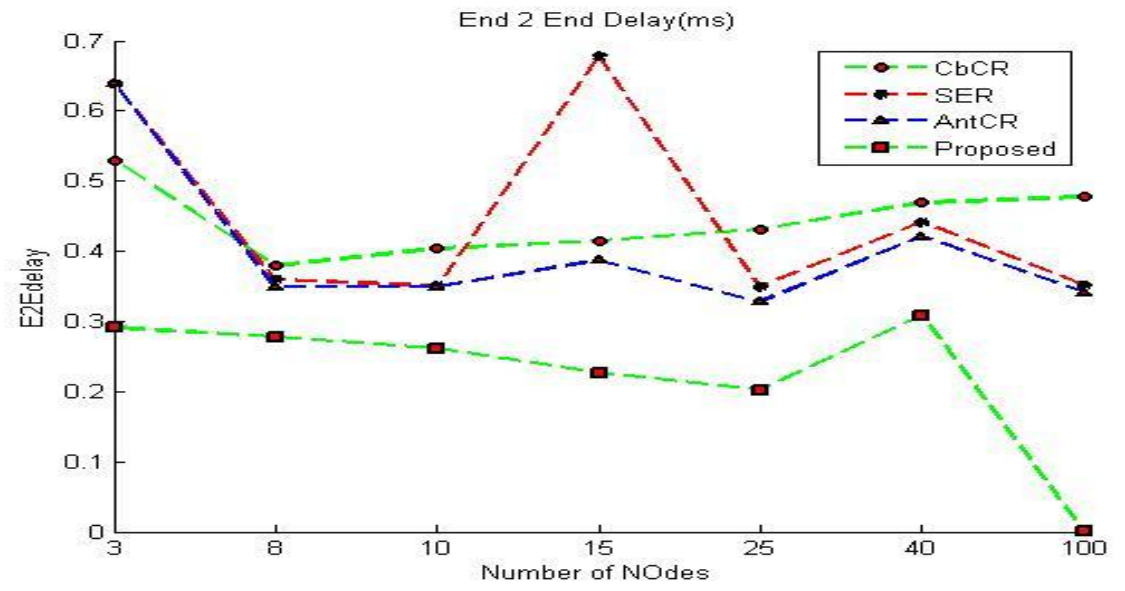

Figure 5: Analysis of E2Edelay

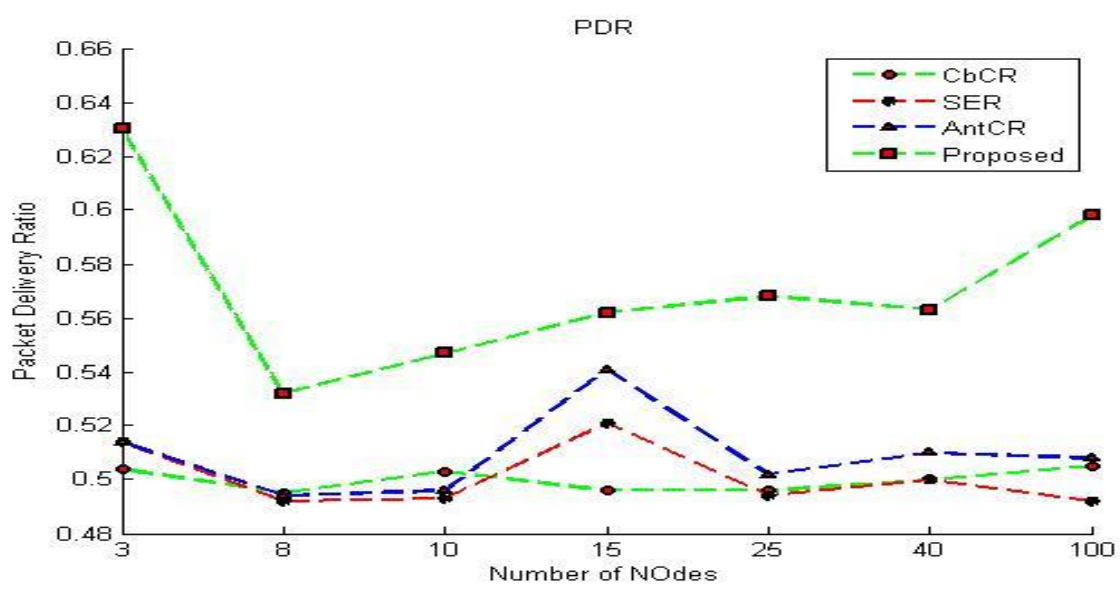

Figure 6: Analysis of Packet Delivery ratio 


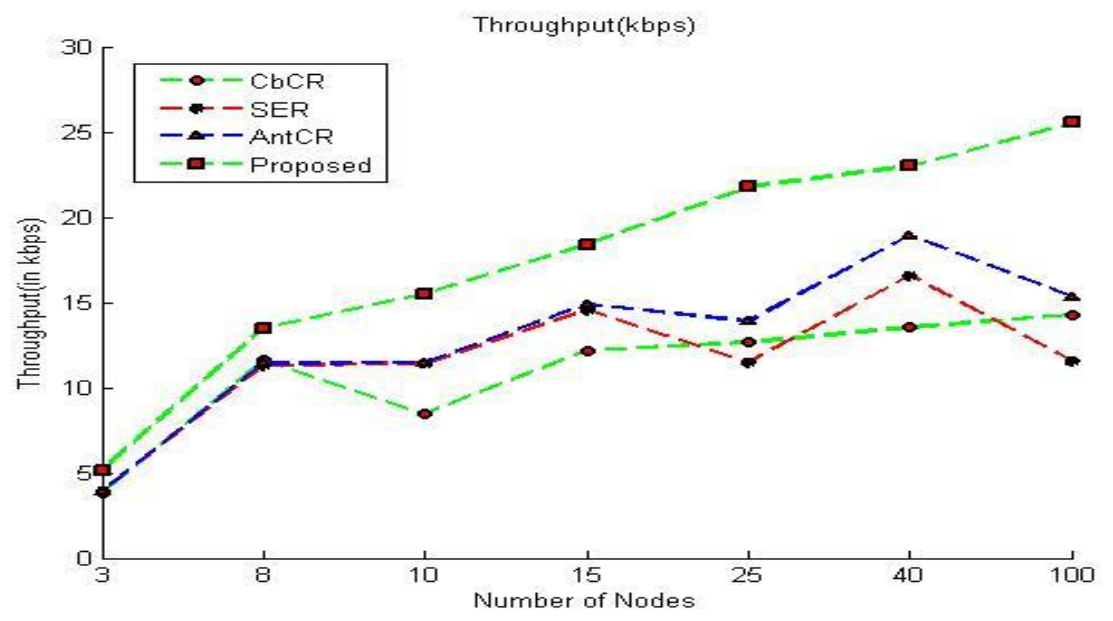

Figure 7: Analysis of Throughput

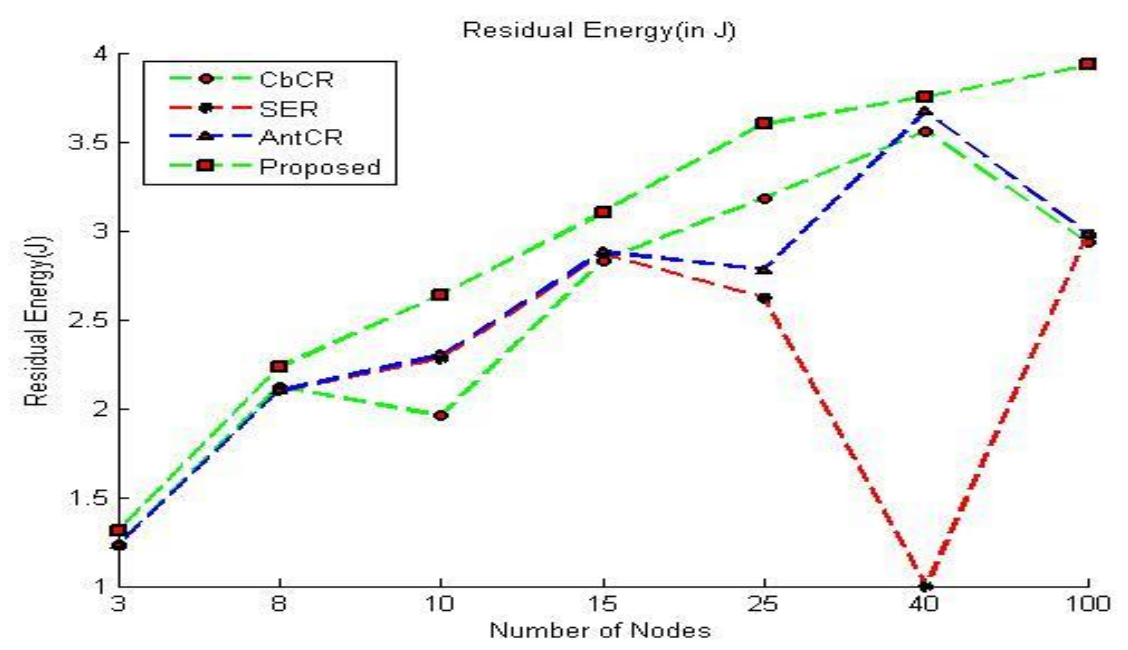

Figure 8: Analysis of Residual Energy

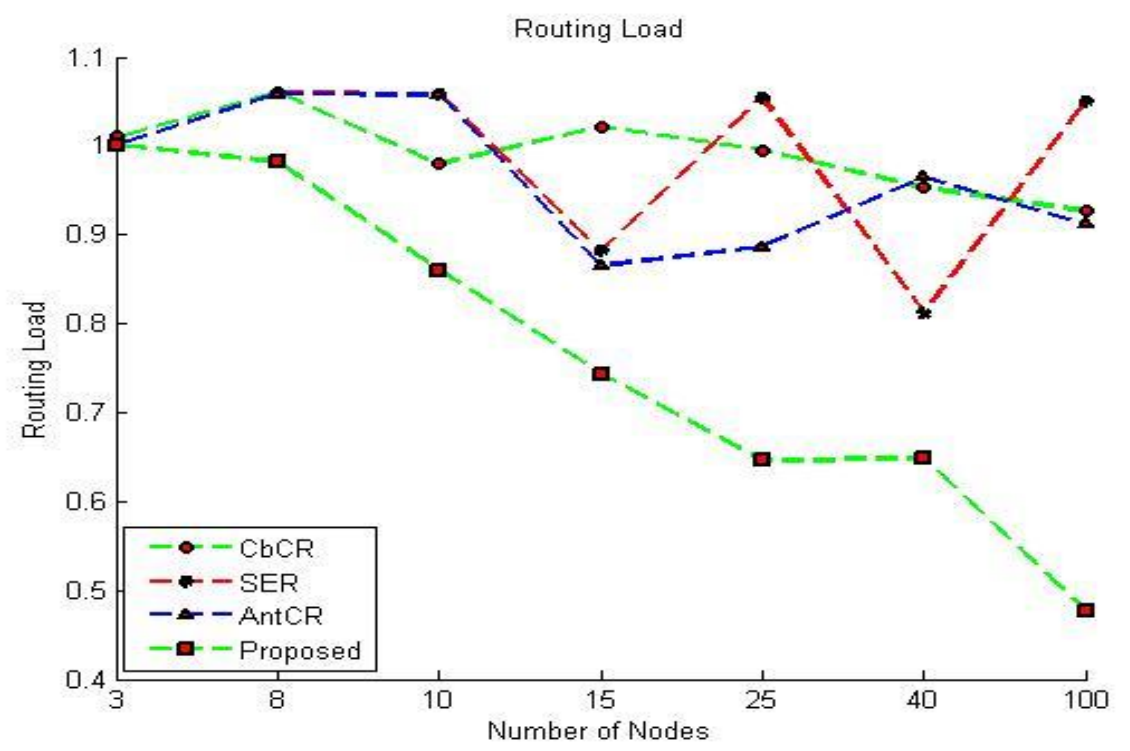

Figure 9: Analysis of Routing load 
The above figures show that the residual energy is more at the same time instance in the modified protocol as compared to the existing protocol. It means the modified chain based protocol consumes less energy as compared to the existing protocol. Moreover the lifetime of the network is approx $10 \%$ greater than the existing protocol. It can be viewed from the figures shown above. Overall we can say that modified chain based cognitive radio protocol is energy efficient as compared to the existing protocol

\section{CONCLUSION}

This paper designs an optimized algorithm based on the ant-lion by using the the hunting behavior of the ant lion. The performance analysis clearly signifies that the algorithm is better in terms of delay, energy consumption as well as throughput as compared to the existing state of art techniques. The performance enhanced is due to the exploration and exploitation search within the search space. In future this work can be extended for other area of applications.

\section{REFERENCES}

[1] Ding, L., Melodia, T., Batalama, S., \& Medley, M. J. (2009, October). Rosa: distributed joint routing and dynamic spectrum allocation in cognitive radio ad hoc networks. In Proceedings of the 12th ACM international conference on Modeling, analysis and simulation of wireless and mobile systems (pp. 13-20). ACM

[2] Fragkiadakis, A. G., Tragos, E. Z., \& Askoxylakis, I. G. "A survey on security threats and detection techniques in cognitive radio networks.", Communications Surveys \& Tutorials, IEEE, 15(1), 428-445. (2013).

[3] Gronsund, P., and O. Grondalen. "Performance Of A Sensor Network Aided Cognitive Radio System." In Personal Indoor and Mobile Radio Communications (PIMRC), 2011 IEEE 22nd International Symposium on, pp. 629-634. IEEE, 2011.

[4] Guan, Quansheng, F. Richard Yu, Shengming Jiang, and Gang Wei. (2010) Prediction-based topology control and routing in cognitive radio mobile ad hoc networks. Vehicular Technology, IEEE Transactions on 59, no. 9 (2010)

[5] Hiren Kumar Deva Sarma et. al. ,An Energy Balanced Routing Protocol for Cognitive Wireless Sensor Networks", Proceedings of the World Congress on Engineering and Computer Science 2013, Vol. II, WCECS 2013, 23-25 October, 2013, San Francisco, USA.

[6] Hou, Y. T., Shi, Y., \& Sherali, H. D. , "Spectrum sharing for multi-hop networking with cognitive radios.", Selected Areas in Communications, IEEE Journal on, 26(1), 146-155. (2008).

[7] Jiang, Xia, and Tracy Camp. "An efficient location server for an ad hoc network."Colorado School Mines, Golden, CO, Tech. Rep., MCS-03-06 (2003).

[8] Janak Kumar Patel, Mitesh Thakkar, “A Survey on Cognitive Radio Wireless Sensor Networks", ISSN: 2321-9939, International Journal Of Engineering Development And Research | Ijedr, Website: Www.Ijedr.Org |

[9] WU Shu-Ci and NIAN Xiao-Hong, "The Architecture and Characteristics of Wireless Sensor network", International Conference on Computer Technology and Development - vol2. No1., pp.561-565, (Dec 2010). 